\title{
Complexity Metrics for Component Based Software - A Comparative Study
}

\author{
Sonal Gehlot ${ }^{1}$, PoojaRana ${ }^{1}$, Rajender Singh ${ }^{2 *}$ \\ ${ }^{1}$ Maharshi Dayanand University, Rohtak, Haryana, India. \\ ${ }^{2}$ Department of Computer Science \& Application, Maharshi Dayanand University, Rohtak, Haryana, India. \\ *Corresponding author: Tel. 08199023624, email: chhillar02@gmail.com \\ Manuscript submitted March 10, 2019; accepted May 20, 2019. \\ doi: $10.17706 /$ jcp.14.6.389-396
}

\begin{abstract}
The Component Based Software Development (CBSD) approach is becoming the trend for software development and is based on developing the software from existing components instead of developing software from the scratch level. Measuring the software complexity is an important aspect during software development as it is an important determinant of software development effort, testing effort, cost, maintainability etc. Interactions/interfaces among components play an important role in contributing complexity to a component based software. In this paper a comparison between different complexity metrics developed by different authors is performed. These metrics are performed by taking different factors to calculate the complexity of the components based software, these factors are instance variables, instance methods, control flow and interface methods etc.. The comparison is performed by taking some quality factors into consideration like maintainability, Integrity, complexity, testability, customizability etc.
\end{abstract}

Key words: CBAD, coupling, weighted assignment technique, complexity metrics, cyclometric complexity, and black box component.

\section{Introduction}

Software metrics play a very important role in assessing and predicting various attributes of software such as complexity, reusability, maintainability, testability etc. Among these attributes complexity affects all other attributes of the software [1]. Software Complexity measures have great importance because it indicates scope of further improvement in software development. Higher value of complexity increases efforts of testing, maintenance and also difficult to reuse.

The component based software development (CBAD) is one of the most important paradigms. CBAD approach is increasingly being adopted for software development. This approach uses reusable components as building blocks for constructing software application. The main aim of this approach is to minimize the development cost, time and efforts by mean of reuse [1].

The major problem faced in Component development is its complexity. So it is necessary to measure the software complexity and reduced it to achieve the maximum benefits of CBAD with minimum cost and efforts. There are several metrics which are available for measuring software complexity but they are not suitable for CBAD.

Software complexity cannot be removed completely but can be controlled only. For controlling of complexity, from time to time many researchers have proposed various metrics for evaluating, predicting and controlling software complexity. Traditional software metrics are usually applicable to small programs, 
whereas the metrics for object-oriented and component based software applications should depend mainly on the granularity and interoperability aspects of the classes and components. The major factor influencing the CBAD is the dependency among software components, which is necessary and desirable because one component may provide the services to another component. Then there should be an interface between the components.

The following figure (Fig. 1) shows the technique for developing software application from existing components (cp).

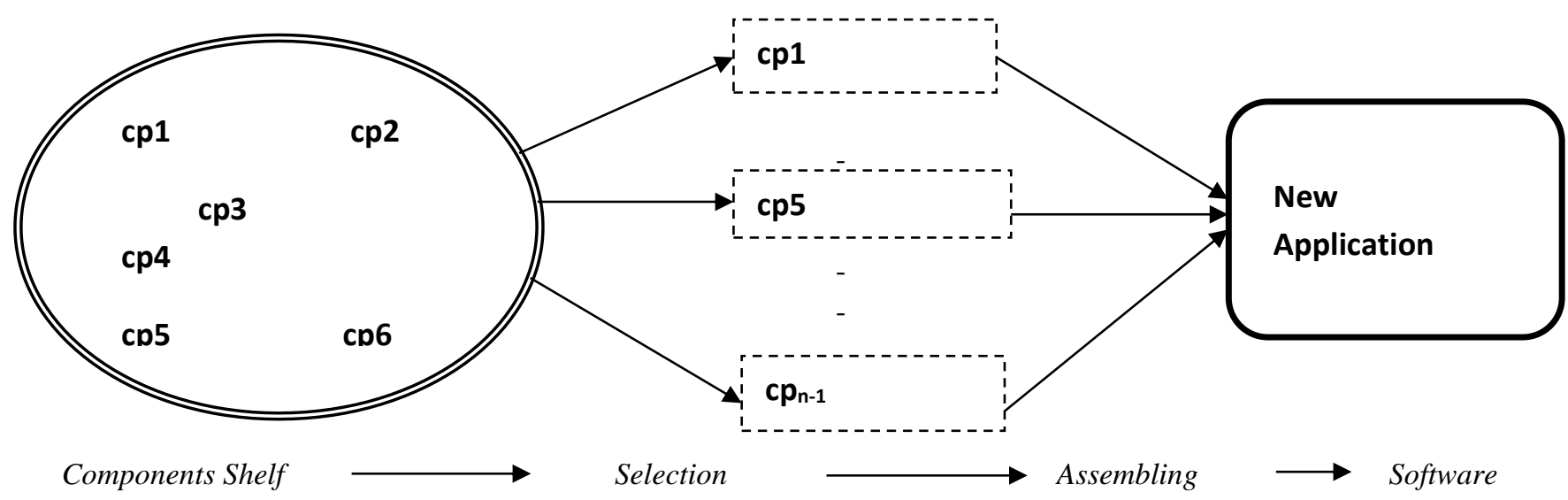

Fig. 1. Component based software development technique.

The paper is divided into sections. First section is introduction; second section has comparison of different complexity metrics. Section 3 has key observation and Section 4 concludes the paper.

\section{Complexity Metrics for Component Based Application}

The dependency among components may be defined as the reliance of a component on others to support a specific functionality or configuration. In CBSE application the components interact with other components by sharing information in order to provide application functionalities. This composition creates interaction that promotes dependencies among components. Application functionalities cannot solely encapsulate within one component. Therefore changing a component may affect that composite functionality, which is reflected in different components. In addition, replacing a new version of a specific component might involve replacing the component on which it depends, in order to preserve a specific application's functionality.

The component complexity closely depends on what contributes to develop the components. Thus there are four elements that affect the component complexity. First element is Variable factor that tells complexity of the variables defined in the component. The variables may consist of member variables of a class having scope for the entire class and the parameters, which are local to a particular method. The second element is interfaces, which are the access points of component, through which a component can request a service declared in an interface of the service providing component.

Interface complexity is defined as sum of complexity of the interface methods of the class. Third element is coupling factor that tells rate of coupling of the methods in the component. Fourth element is cyclometic complexity of the methods of the component.

In this paper we will review the different types of complexity metrics and compare them.

Nael SALMAN et al. [2] 2006 author in this paper developed several complexity metrics for component based system. The main focus of the author is to find out the strength of the software, by defining metrics on its structural complexity [2]. The main factors that determine the complexity are components, connectors and composition tree. 
For components three metrics are defined such as TNC, ANMC and TNIC, for connectors three metrics are developed like TNL, ANLC, ANLI [2]. the author defined a suite of metrics for component based software and the definition of component based software is adopted by author which is given by Szyperski (1999) [2].

Gill and Balkishan et al. [3] 2008 their attempt was to identify the impact of dependency among components on software complexity. They proposed two metrics CDM and CIDM. Both the metrics can be applied once the directed graph and adjacency matrix of the design is found [3]. The result of these metrics is analyzed in order to determine as how the interaction among components and number of components affect the complexity of component based application. These metric shows higher interaction between components increases the complexity because of more coupling among component. Higher complexity means more expensive software and less maintainability [3].

Gui Gui and Paul. D. Scott et al. [4] 2009 in this paper the author develop new metrics for coupling and cohesion to measure the reusability of a component. These metrics are different from other metrics in three aspects, first is degree of resemblance of one component with other component, degree of coupling, direct coupling and cohesion relationship. Author measures quantitatively measure the complexity by taking these factors into consideration [4]. A comparative analysis is performed between new metrics and existing metrics and found that new metrics are much superior then existing metrics [4].

Narasimhan and Hendradjaya's metric suite et al. [5] 2009 in this paper author defined a metric suite for component based software. Author defined two sets of metrics for measuring complexity and criticality. First set of metrics are Component Packing Density (CPD) which describe the binding of the components. These metrics relate all component constituents to the number of integrated component. Another set of metrics are Component Interaction Density (CID), these metrics relates interaction between components and available number of interaction in the system [5].

Kumari and Bhasin et al. [6] 2011 the authors aimed to design a composite complexity measure to quantify important aspects of complexity of a component based application. Two major complexity metric of CBS are one due to individual component named as TC (CBS) and other due to its interaction with other component named as IACC [6]. They take different factors like size, type of variables, nesting level of control structure to calculate the individual component complexity. Graph theoretic notions and concept of weights have been used for interface component complexity. The result shows that the effect of these parameters (Size, Nesting Structure, Control Structure etc.) on complexity of a CBS is quite significant. The results also show that higher interaction between components increases the complexity because of more coupling among the components [6].

Table 1. Metrics of Different Authors and Comparisons

\begin{tabular}{|c|c|c|c|c|c|}
\hline Sr.No & Metric & Formula Used & Description & Author/s & $\begin{array}{c}\text { External } \\
\text { Quality } \\
\text { Attributes } \\
\end{array}$ \\
\hline 1. & $\begin{array}{l}\text { Total No of } \\
\text { Components } \\
\text { (TNC) [2] }\end{array}$ & $\begin{array}{l}\mathrm{TNC}=\text { Count of all components } \\
\text { in the system [2] }\end{array}$ & $\begin{array}{l}\text { The count of all components } \\
\text { in the system that appear in } \\
\text { different levels of } \\
\text { abstractions [2]. }\end{array}$ & $\begin{array}{l}\text { SALMAN et.al. } \\
\text { (2006) [2] }\end{array}$ & $\begin{array}{l}\text { Integrability } \\
\text { and } \\
\text { maintainability }\end{array}$ \\
\hline 2. & $\begin{array}{l}\text { Average no of } \\
\text { methods per } \\
\text { component(A } \\
\text { NMC) [2] }\end{array}$ & $\begin{array}{l}\text { ANMC=total no of methods/ } \\
\text { total no of component [2] }\end{array}$ & $\begin{array}{l}\text { This metrics is estimated by } \\
\text { dividing the total number of } \\
\text { methods by the total number } \\
\text { of components [2]. }\end{array}$ & $\begin{array}{l}\text { SALMAN et.al. } \\
\text { (2006) [2] }\end{array}$ & $\begin{array}{l}\text { Integrability } \\
\text { and } \\
\text { maintainability }\end{array}$ \\
\hline 3. & $\begin{array}{l}\text { Total no of } \\
\text { links (TNI) [2] }\end{array}$ & $\mathrm{TNI}=$ count of all links [2] & $\begin{array}{l}\text { Count of all links appearing } \\
\text { in the system design model } \\
\text { in all levels [2]. }\end{array}$ & $\begin{array}{l}\text { SALMAN et.al. } \\
\text { (2006) [2] }\end{array}$ & $\begin{array}{l}\text { Integrability } \\
\text { and } \\
\text { maintainability }\end{array}$ \\
\hline Sr.No & Metric & Formula Used & Description & Author/s & $\begin{array}{l}\text { External } \\
\text { Quality } \\
\text { Attributes } \\
\end{array}$ \\
\hline
\end{tabular}




\begin{tabular}{|c|c|c|c|c|c|}
\hline 4. & $\begin{array}{l}\text { Average } \\
\text { number of } \\
\text { links between } \\
\text { components } \\
\text { (ANLC) [2] } \\
\end{array}$ & $\begin{array}{l}\text { ANLC= Total no of links / Total } \\
\text { no of components [2] }\end{array}$ & $\begin{array}{l}\text { Total number of links } \\
\text { divided by the total number } \\
\text { of components [2]. }\end{array}$ & $\begin{array}{l}\text { SALMAN et.al. } \\
(2006)[2]\end{array}$ & $\begin{array}{l}\text { Integrability } \\
\text { and } \\
\text { maintainability }\end{array}$ \\
\hline 5. & $\begin{array}{l}\text { Average } \\
\text { number } \\
\text { links per } \\
\text { interface } \\
\text { (ANLI) [2] } \\
\end{array}$ & $\begin{array}{l}\text { ANLI }=\text { Total no of links } \\
\text { between interfaces / Total no } \\
\text { of interfaces [2] }\end{array}$ & $\begin{array}{l}\text { Total number of links } \\
\text { between interfaces divided } \\
\text { by the total number of } \\
\text { interfaces [2]. }\end{array}$ & $\begin{array}{l}\text { SALMAN et.al. } \\
(2006)[2]\end{array}$ & $\begin{array}{l}\text { Integrability } \\
\text { and } \\
\text { maintainability }\end{array}$ \\
\hline 6. & $\begin{array}{l}\text { Total number } \\
\text { of interfaces } \\
\text { (TNI) [2] }\end{array}$ & $\begin{array}{l}\text { TNI=count of all interfaces in } \\
\text { all components [2] }\end{array}$ & $\begin{array}{l}\text { Count of all interfaces of all } \\
\text { Components in the system } \\
{[2] .}\end{array}$ & $\begin{array}{l}\text { SALMAN et.al. } \\
\text { (2006) [2] }\end{array}$ & $\begin{array}{l}\text { Integrability } \\
\text { and } \\
\text { maintainability }\end{array}$ \\
\hline 7. & $\begin{array}{l}\text { Average } \\
\text { number of } \\
\text { interfaces per } \\
\text { component } \\
\text { (ANIC) [2] }\end{array}$ & $\begin{array}{l}\text { ANIC= Total no of interfaces/ } \\
\text { Total no of components [2] }\end{array}$ & $\begin{array}{l}\text { Total number of interfaces } \\
\text { divided by the total number } \\
\text { of components [2]. }\end{array}$ & $\begin{array}{l}\text { SALMAN et.al. } \\
(2006)[2]\end{array}$ & $\begin{array}{l}\text { Integrability } \\
\text { and } \\
\text { maintainability }\end{array}$ \\
\hline 8. & $\begin{array}{l}\text { Depth of the } \\
\text { composition } \\
\text { tree (DCT) [2] }\end{array}$ & $\begin{array}{l}\text { DCT }=\text { Count of the number of } \\
\text { levels [2] }\end{array}$ & $\begin{array}{l}\text { Count the number of levels } \\
\text { of the composition tree [2]. }\end{array}$ & $\begin{array}{l}\text { SALMAN et.al. } \\
(2006)[2]\end{array}$ & $\begin{array}{l}\text { Integrability } \\
\text { and } \\
\text { maintainability }\end{array}$ \\
\hline 9. & $\begin{array}{l}\text { Component } \\
\text { Dependency } \\
\text { Metric (CDM) } \\
{[3]}\end{array}$ & $\begin{array}{l}\mathrm{CDM}=\sum_{j=1}^{n} D j \\
\mathrm{Dj}=\sum_{j=1}^{n} \operatorname{path}(i, j)[3]\end{array}$ & $\begin{array}{l}\text { The complexity results from } \\
\text { dependencies among } \\
\text { application components. } \\
\text { Dependency of a component } \\
\mathrm{C}_{\mathrm{i}} \text { to other component is the } \\
\text { number of all the paths in the } \\
\text { graph from } \mathrm{C}_{\mathrm{i}} \text { to the other } \\
\text { component [3]. }\end{array}$ & $\begin{array}{l}\text { Gill and } \\
\text { Balkishan et. } \\
\text { al. (2008) [3] }\end{array}$ & $\begin{array}{l}\text { Complexity } \\
\text { Maintainability }\end{array}$ \\
\hline 10 & $\begin{array}{l}\text { Component } \\
\text { Interaction } \\
\text { Density } \\
\text { Metric } \\
\text { (CIDM)[3] }\end{array}$ & $\operatorname{CIDM} \frac{I}{N}=[3]$ & $\begin{array}{l}\text { This Metric computes the } \\
\text { ratio of total number of } \\
\text { direct interactions between } \\
\text { the components to the total } \\
\text { number of components [3]. }\end{array}$ & $\begin{array}{l}\text { Gill and } \\
\text { Balkishan et. } \\
\text { al. (2008) [3] }\end{array}$ & $\begin{array}{l}\text { Complexity } \\
\text { Maintainability }\end{array}$ \\
\hline 11 & $\begin{array}{l}\text { Direct } \\
\text { Coupling [4] }\end{array}$ & $\operatorname{CoupD}(i, j)=\frac{\left|M V_{i, j}\right|}{\left|M V_{i}\right|+\left|V_{i}\right|+\left|M_{i}\right|}$ & $\begin{array}{l}\text { It measures the direct } \\
\text { coupling } \\
\text { Components [4]. }\end{array}$ & $\begin{array}{l}\text { Gui and Paul } \\
\text { et.al. (2009) } \\
\text { [4] }\end{array}$ & $\begin{array}{l}\text { Complexity } \\
\text { Reusability }\end{array}$ \\
\hline 12 & $\begin{array}{l}\text { Transitive } \\
\text { Coupling [4] }\end{array}$ & $\begin{array}{l}\operatorname{CoupT}(i, j, p)=\prod_{e_{s, t} \in p} \operatorname{CoupD}(s, t) \\
=\prod_{e_{s, t} \in p} \frac{\left|M V_{s, t}\right|}{\left|M V_{s}\right|+\left|V_{s}\right|+\left|M_{s}\right|}\end{array}$ & $\begin{array}{l}\text { It measures the transitive } \\
\text { coupling between classes } \mathrm{Ci} \\
\text { and } \mathrm{Cj} \text { due to a specific path } \\
\mathrm{P}[4] \text {. }\end{array}$ & $\begin{array}{l}\text { Gui and Paul } \\
\text { et.al. (2009) } \\
{[4]}\end{array}$ & $\begin{array}{l}\text { Complexity } \\
\text { Reusability }\end{array}$ \\
\hline 13 & $\begin{array}{l}\text { Weighted } \\
\text { Transitive } \\
\text { Coupling [4] }\end{array}$ & WTCoup $=\frac{\sum_{i, j=1}^{n} \operatorname{Coup}(i, j)}{m^{2}-m}$ & $\begin{array}{l}\text { It measures the total } \\
\text { coupling of the software } \\
\text { system [4]. }\end{array}$ & $\begin{array}{l}\text { Gui and Paul } \\
\text { et.al. (2009) } \\
{[4]}\end{array}$ & $\begin{array}{l}\text { Complexity } \\
\text { Reusability }\end{array}$ \\
\hline 14 & $\begin{array}{l}\text { Transitive } \\
\text { Cohesion of } \\
\text { the class [4] }\end{array}$ & $\begin{array}{l}\text { ClassCohT }=\frac{\sum_{i, j=1}^{m} \operatorname{Sim}(i, j)}{m^{2}-m} \\
{[4]}\end{array}$ & $\begin{array}{l}\text { It measures the cohesion of } \\
\text { class by summing the } \\
\text { similarities of all methods } \\
\text { and dividing by total number } \\
\text { of methods [4]. }\end{array}$ & $\begin{array}{l}\text { Gui and Paul } \\
\text { et.al. (2009) } \\
{[4]}\end{array}$ & $\begin{array}{l}\text { Complexity } \\
\text { Reusability }\end{array}$ \\
\hline 15 & $\begin{array}{l}\text { Intransitive } \\
\text { Cohesion of } \\
\text { class [4] }\end{array}$ & $\begin{array}{l}\text { ClassCohD }=\frac{\sum_{i, j=1}^{m} \operatorname{Sim} D(i, j)}{m^{2}-m} \\
{[4]}\end{array}$ & $\begin{array}{l}\text { It measures the direct } \\
\text { cohesion } \\
\text { components [4]. }\end{array}$ & $\begin{array}{l}\text { Gui and Paul } \\
\text { et.al. (2009) } \\
\text { [4] }\end{array}$ & $\begin{array}{l}\text { Complexity } \\
\text { Reusability }\end{array}$ \\
\hline 16 & $\begin{array}{l}\text { Weighted } \\
\text { Transitive } \\
\text { Cohesion [4] }\end{array}$ & WTCoh $=\frac{\left.\sum_{j=1}^{n} \text { ClassCohT } j\right)}{n}[4]$ & $\begin{array}{l}\text { It measures weighted } \\
\text { transitive cohesion. The } \\
\text { value of WTCoh should lie } \\
\text { between } 0 \text { to } 1[4] .\end{array}$ & $\begin{array}{l}\text { Gui and Paul } \\
\text { et.al. (2009) } \\
{[4]}\end{array}$ & $\begin{array}{l}\text { Complexity } \\
\text { Reusability }\end{array}$ \\
\hline 17 & $\begin{array}{l}\text { Weighted } \\
\text { Intransitive } \\
\text { Cohesion [4] }\end{array}$ & WICoh $=\frac{\left.\sum_{j=1}^{n} \operatorname{ClassCohD} j\right)}{n}[4]$ & $\begin{array}{l}\text { It measures weighted } \\
\text { intransitive cohesion. The } \\
\text { value of WICoh should lie } \\
\text { between } 0 \text { to } 1 \text { [4]. }\end{array}$ & $\begin{array}{l}\text { Gui and Paul } \\
\text { et.al. (2009) } \\
{[4]}\end{array}$ & $\begin{array}{l}\text { Complexity } \\
\text { Reusability }\end{array}$ \\
\hline Sr.No & Metric & Formula Used & Description & Author/s & $\begin{array}{c}\text { External } \\
\text { Quality } \\
\text { Attributes } \\
\end{array}$ \\
\hline
\end{tabular}




\begin{tabular}{|c|c|c|c|c|c|}
\hline 18 & $\begin{array}{l}\text { Component } \\
\text { Packing } \\
\text { Density [5] }\end{array}$ & $\begin{array}{l}\text { CPD } \text { constituent_type }_{\text {\# }}= \\
\frac{\# \text { Constituent }>}{\# \text { Compoenent }}[5]\end{array}$ & $\begin{array}{l}\text { It is a ratio between Number } \\
\text { of constituents and Number } \\
\text { of components [5]. }\end{array}$ & $\begin{array}{l}\text { Narasimhan } \\
\text { et.al. (2009) } \\
{[5]}\end{array}$ & $\begin{array}{l}\text { Complexity } \\
\text { Criticality }\end{array}$ \\
\hline 19 & $\begin{array}{l}\text { Interaction } \\
\text { Density } \\
\text { Metric [5] }\end{array}$ & $\mathrm{IDC}=\frac{\# I}{\# \operatorname{Imax}}[5]$ & $\begin{array}{l}\text { It is a ratio between actual } \\
\text { interaction and available } \\
\text { interaction [5]. }\end{array}$ & $\begin{array}{l}\text { Narasimhan } \\
\text { et.al. (2009) } \\
\text { [5] }\end{array}$ & $\begin{array}{l}\text { Complexity } \\
\text { Interdependenc } \\
\text { e } \\
\text { Interface } \\
\end{array}$ \\
\hline 20 & $\begin{array}{l}\text { Incoming } \\
\text { Interaction } \\
\text { Density of } \\
\text { Component } \\
\text { [5] }\end{array}$ & $\mathrm{IIDC}=\frac{\# I_{I N}}{\# \operatorname{Imax} I N}[5]$ & $\begin{array}{l}\text { It is a ratio between number } \\
\text { of incoming interaction used } \\
\text { and available number of } \\
\text { incoming interaction [5]. }\end{array}$ & $\begin{array}{l}\text { Narasimhan } \\
\text { et.al. (2009) } \\
{[5]}\end{array}$ & $\begin{array}{l}\text { Complexity } \\
\text { Interdependenc } \\
\text { e } \\
\text { Interface }\end{array}$ \\
\hline 21 & $\begin{array}{l}\text { Outgoing } \\
\text { Interaction } \\
\text { Density of } \\
\text { Components } \\
\text { [5] }\end{array}$ & OIDC $=\frac{\# I_{\text {OUT }}}{\# \operatorname{Imax}_{\text {OUT }}}[5]$ & $\begin{array}{l}\text { It is a ratio between number } \\
\text { of outgoing interaction used } \\
\text { and available number of } \\
\text { outgoing interaction [5]. }\end{array}$ & $\begin{array}{l}\text { Narasimhan } \\
\text { et.al. (2009) } \\
{[5]}\end{array}$ & $\begin{array}{l}\text { Complexity } \\
\text { Interdependenc } \\
\text { e } \\
\text { Interface }\end{array}$ \\
\hline 22 & $\begin{array}{l}\text { Average } \\
\text { Interaction } \\
\text { Density } \\
\text { Metric [5] }\end{array}$ & $\mathrm{AIDC}=\frac{I D C 1+I D C 2+\cdots+I D C n}{\# \text { Components }}[5]$ & $\begin{array}{l}\text { It is a sum of each } \\
\text { components interaction } \\
\text { density and divided by total } \\
\text { number of components [5]. }\end{array}$ & $\begin{array}{l}\text { Narasimhan } \\
\text { et.al. (2009) } \\
\text { [5] }\end{array}$ & $\begin{array}{l}\text { Complexity } \\
\text { Interdependenc } \\
\text { e } \\
\text { Interface }\end{array}$ \\
\hline 23 & $\begin{array}{l}\text { Total } \\
\text { Complexity of } \\
\text { a Component } \\
\text { Based System } \\
\text { (TC(CBS)) [6] }\end{array}$ & $\begin{array}{l}\mathrm{TC}(\mathrm{CBS})=\quad \sum_{j=1}^{n}((I C C) j * \\
(W t) j) *(I A C C) j[6]\end{array}$ & $\begin{array}{l}\text { This composite metric takes } \\
\text { different attributes of } \\
\text { complexity. The result shows } \\
\text { the effect of these } \\
\text { parameters on complexity of } \\
\text { a CBS [6]. }\end{array}$ & $\begin{array}{l}\text { Kumari and } \\
\text { Bhasin et.al. } \\
(2011)[6]\end{array}$ & $\begin{array}{l}\text { Complexity } \\
\text { Testing } \\
\text { Maintainability }\end{array}$ \\
\hline 24 & $\begin{array}{l}\text { Interaction } \\
\text { Among } \\
\text { Components } \\
\text { Complexity(I } \\
\text { ACC) [6] }\end{array}$ & IACC $=$ IIC+OIC [6] & $\begin{array}{l}\text { This metric shows the } \\
\text { interaction with other } \\
\text { component. The concept of } \\
\text { link is used to quantify } \\
\text { interface aspect of a } \\
\text { component [6]. }\end{array}$ & $\begin{array}{l}\text { Kumari and } \\
\text { Bhasin et.al. } \\
(2011)[6]\end{array}$ & $\begin{array}{l}\text { Complexity } \\
\text { Testing } \\
\text { Maintainability }\end{array}$ \\
\hline 25 & $\begin{array}{l}\text { Average } \\
\text { Incoming } \\
\text { Interactions } \\
\text { Complexity } \\
\text { (AIIC) [7] }\end{array}$ & $\mathrm{AIIC}=\frac{\sum_{i=1}^{m} I I i}{m}[7]$ & $\begin{array}{l}\text { This metric shows the } \\
\text { average of the incoming } \\
\text { interactions of one } \\
\text { component [7]. }\end{array}$ & $\begin{array}{l}\text { Kumari and } \\
\text { Upadhyaya et. } \\
\text { al. (2011) [7] }\end{array}$ & $\begin{array}{l}\text { Complexity } \\
\text { Reliability }\end{array}$ \\
\hline 26 & $\begin{array}{l}\text { Average } \\
\text { Outgoing } \\
\text { Interactions } \\
\text { Complexity } \\
\text { (AOIC) [7] }\end{array}$ & $\mathrm{AOIC}=\frac{\sum_{i=1}^{m} O I i}{m}[7]$ & $\begin{array}{l}\text { This metric shows the } \\
\text { average of the outgoing } \\
\text { interactions of one } \\
\text { component [7]. }\end{array}$ & $\begin{array}{l}\text { Kumari and } \\
\text { Upadhyaya et. } \\
\text { al. (2011) [7] }\end{array}$ & $\begin{array}{l}\text { Complexity } \\
\text { Reliability }\end{array}$ \\
\hline 27 & $\begin{array}{l}\text { Average } \\
\text { Interface } \\
\text { Complexity of } \\
\text { a Component } \\
\text { Based System } \\
\text { (AIC(CBS)) } \\
{[7]}\end{array}$ & $\begin{array}{l}\mathrm{AIC}(\mathrm{CBS})= \\
\frac{\sum_{i=1}^{m} I I i}{m}+\frac{\sum_{i=1}^{m} O I i}{m}[7]\end{array}$ & $\begin{array}{l}\text { This Metric shows the } \\
\text { average interface metric by } \\
\text { summation of incoming } \\
\text { interface and outgoing } \\
\text { interface metrics [7]. }\end{array}$ & $\begin{array}{l}\text { Kumari and } \\
\text { Upadhyaya } \\
\text { et.al. (2011) } \\
\text { [7] }\end{array}$ & $\begin{array}{l}\text { Complexity } \\
\text { Reliability }\end{array}$ \\
\hline 28 & $\begin{array}{l}\text { Complexity of } \\
\text { Interface } \\
\text { Component } \\
\text { (CI) [8] }\end{array}$ & $\begin{array}{l}\mathrm{CI}= \\
\mathrm{CSI}+\mathrm{ICC}(\text { internal })+\mathrm{ICC}(\text { extern } \\
\text { al) }[8]\end{array}$ & $\begin{array}{l}\text { This metric measured in } \\
\text { term of size of interface, } \\
\text { interface coupling with } \\
\text { internal subcomponents and } \\
\text { interface coupling with outer } \\
\text { components [8]. }\end{array}$ & $\begin{array}{l}\text { Chillar and } \\
\text { Ahlawat et.al. } \\
\text { (2012) [8] }\end{array}$ & $\begin{array}{l}\text { Complexity } \\
\text { Reusability } \\
\text { Customizability }\end{array}$ \\
\hline 29 & $\begin{array}{l}\text { Average } \\
\text { Complexity of } \\
\text { CBS [8] }\end{array}$ & $\mathrm{AC}(\mathrm{CBS})=\sum_{i=1}^{n} C I / n[8]$ & $\begin{array}{l}\text { This complexity measured } \\
\text { the average complexity of } \\
\text { interface component [8]. }\end{array}$ & $\begin{array}{l}\text { Chillar and } \\
\text { Ahlawat et. al. } \\
\text { (2012) [8] }\end{array}$ & $\begin{array}{l}\text { Complexity } \\
\text { Reusability } \\
\text { Customizability }\end{array}$ \\
\hline
\end{tabular}




\begin{tabular}{|c|c|c|c|c|c|}
\hline Sr.No & Metric & Formula Used & Description & Author/s & $\begin{array}{c}\text { External } \\
\text { Quality } \\
\text { Attributes } \\
\end{array}$ \\
\hline 30 & $\begin{array}{l}\text { Interface } \\
\text { Dependency } \\
\text { Metric (IDM) } \\
\text { [8] }\end{array}$ & $\begin{array}{l}\text { IDM = Functionality obtained } \\
\text { from other } \\
\text { component/functionality of a } \\
\text { component [8] }\end{array}$ & $\begin{array}{l}\text { The dependency of a } \\
\text { component can be as the } \\
\text { functionality obtained from } \\
\text { other components apart } \\
\text { from its own functionality } \\
\text { [8]. }\end{array}$ & $\begin{array}{l}\text { Chillar and } \\
\text { Ahlawat et.al. } \\
\text { (2012) [8] }\end{array}$ & $\begin{array}{l}\text { Complexity } \\
\text { Reusability } \\
\text { Customizability }\end{array}$ \\
\hline 31 & $\begin{array}{l}\text { Average } \\
\text { interface } \\
\text { dependency } \\
\text { metric of CBS } \\
\text { [8] }\end{array}$ & $\operatorname{AIDM}(\mathrm{CBS})=\sum_{i=0}^{n} I D M / n[8]$ & $\begin{array}{l}\text { This metric measured the } \\
\text { average complexity of } \\
\text { interface dependency metric } \\
\text { [8]. }\end{array}$ & $\begin{array}{l}\text { Chillar and } \\
\text { Ahlawat et.al. } \\
\text { (2012) [8] }\end{array}$ & $\begin{array}{l}\text { Complexity } \\
\text { Reusability } \\
\text { Customizability }\end{array}$ \\
\hline
\end{tabular}

Kumari and Upadhyaya et al. [7] 2011 they attempted to design an interface complexity metric for black box components to quantify an important aspect of complexity of a CBS. In CBAD a component is linked with other component and has interfaces with them. A link means that a component submits an event and other component receive it. Interface between two components can be through incoming and outgoing interactions. They proposed AIIC, AOIC and AIC (CBS) which calculate average incoming interaction complexity, average outgoing interactions complexity and average interface complexity of a component based application [7]. The result shows that the effect of interface parameter on complexity of CBS is quite significant. They propose that average no of interaction per component in CBS should not be greater than five otherwise that CBS would be highly complex and will be more prone to errors and hence unreliable [7].

Chillar and Ahlawat et al. [8] (2012) they proposed two metrics CI and IDM for measuring complexity of interface and interface dependency of CBS. These metrics are based on different constituents of an interface like interface methods and interface variables with different weights assigned to them. Strength of proposed metrics is computed using weighted assignment technique. Interface methods are classified according to data type of return type and data type of arguments [8]. These metrics shows that higher dependency among components increases complexity because of more coupling. There is a positive relation between complexity of interface metric and interface dependency metric. It is clear that complexity of interface and dependency of interface increases with increase in parameter involved [8].

\section{Key Observations}

Conducted a systematic study of the literature available for the interface metrics for component based applications. These included research publications involving validations, proposals and all other studies related to interface metrics [9]. The search for relevant publications was conducted through various ACM journals. Reference checking was used to make sure that no relevant work was being left out [9]. The contribution of different interface metrics based on the mapping level addressed by them was also studied, which revealed that most of the interface metrics proposed to measure the interdependence of different component using interface component [9].

Further analysis shows that most of the interface metrics address the maintainability, reusability and testability quality factors. Hence a lot of work is still left to be done to prove these metrics are good indicators of the overall software quality.

\section{Conclusion and Future Scope of Work}

This paper provides a thorough survey of interface metrics for Component Based application. The survey conducted covers all the aspects of Interface Metrics for CBS and presents them in categorical form. From the study it was observed that only a limited amount of work has been done in the field [9]. The other main findings along with the possible future directions are. 
- Most of the interface metrics studied lack of validations which limits their usefulness.

- The relationship of these metrics with external quality attributes was also studied and it was found that most of the interface metrics proposed share a relationship with maintainability, testability and reusability.

- Interface Metrics need to be evaluated for a wide range of large scale real world applications for both metric validation and effective utilization for software quality assessment.

The overall study revealed that the interface complexity metrics domain is still has a scope in the field of software engineering and faces a number of research challenges in term of empirical validation and relationship with external software quality attributes. For the future research work, researchers can use these metrics for indirect coupling measure as well as indicator for predicting the various qualities attributes like maintainability, testability and reusability of component based software application.

\section{References}

[1] Kozaczynski, W., \& Booch, G. (1998). Component-based software engineering. IEEE Software, 155, 34-36.

[2] Noel, S. (2006). Complexity metrics as predictors of maintainability and integrability of software components. Journal of Arts and Sciences, 5, 39-50.

[3] Gill, N. S. B. (2008). Dependency and interaction oriented complexity metrics of component-based systems. ACM SIGSOFT Software Engineering Notes, 33(2), 1-5.

[4] Gui, S. (2008). New coupling and cohesion metrics for evaluation of software component reusability. Proceedings of the 9th International Conference For Young Computer Scientists.

[5] Narasimhan,V. L., Parthasarathy, P. T., \& Das, M. (2009). Evaluation of a suite of metrics for component based software engineering (CBSE). Issues in Informing Science and Information Technology, 6.

[6] UshaChhillar, S. (2011). A journey of software metrics: Traditional to aspect-oriented paradigm. Proceedings of the 5th National Conference on Computing for Nation Development (pp. 289-293). New Delhi.

[7] Usha, K., \& Shuchita, U. (2011). An interface complexity measure for component-based software systems. International Journal of Computer Applications (0975-8887), 36(1).

[8] Rajender, S. C., Priyanka, A., \& Usha, K. (2012). Measuring complexity of component based system using weighted assignment technique. Proceedings of the 2nd International Conference on Information Communication and Management (ICICM 2012).

[9] Rani, G., \& Paramvir, S. (2014). Dynamic coupling metrics for object oriented software systems- A survey. ACM SIGSOFT Software Engineering Notes, 39(2).

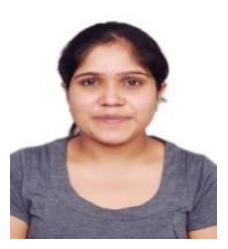

Sonal Gahlot is an assistant professor in DPG College of Engineering since April 2016. she has completed her B.E in computer science and engineering from Gurgaon Institute of Technology and Management, Gurgaon in 2009. Tech in computer science and technology from ITM University with First Division in 2011, completed pre-Ph.D course work from MDU, Rohtak with first division in August 2013 and pursuing Ph.D from MDU, Rohtak. Her areas of interest are software engineering, component-based software engineering, data structures, computer networks.

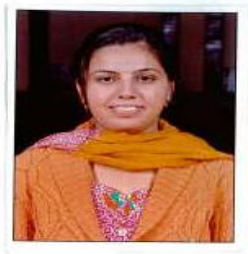

Pooja Rana is an associate professor in Department of Computer Science and Applications, DAV Institute of Management, Faridabad, Haryana, India; and pursuing Ph.D in computer science from Faculty of Computer Science and Applications, M.D. University, Rohtak, Haryana, India. She holds a master of computer application (MCA) from M.D. University, 
Rohtak, India. She obtained her master of technology degree in Information Technology from AAI-DU, Allahabad, India.

Her research interests include software process reengineering, software engineering, software reuse, software process customization and automation, and software process metrics. She has presented and published various papers in international and national journals/conferences.

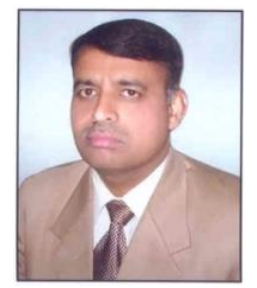

Rajender Singh Chhillar is working as a professor and head of Department of Computer Science and Applications, Maharshi Dayanand University (MDU), Rohtak, Haryana, India. He was the member of monitoring committee of campus wide Networking, M. D. University, Rohtak. He obtained his Ph.D in computer science from Maharishi Dayanand University, Rohtak and master degree from Kurukshetra University, Kurukshetra, Haryana.

Dr. Chhillar's research areas include software engineering, software testing, computer network security, software metrics, component and aspect based metrics, data warehousing and data mining, information and network security and IT management. He has published more than 150 publications in international and national journals/ conferences. Professor Chhillar has also authored two books: Software Engineering: Metrics, Testing and Faults, Excel Books House, New Delhi, Application of Information Technology to Business, Ramesh Books House, Jaipur. 